\title{
FIXED POINT THEOREMS IN COMPLEX VALUED FUZZY $b$-METRIC SPACES WITH APPLICATION TO INTEGRAL EQUATIONS
}

\author{
IZZETTIN DEMIR \\ Received 20 December, 2019
}

\begin{abstract}
In this paper, firstly, we introduce the concept of a complex valued fuzzy $b$-metric space, which is inspired by the work of Shukla et al. [24]. Also, we investigate some of its topological properties which strengthen this concept. Next, we establish some fixed point theorems in the context of complex valued fuzzy $b$-metric spaces and give suitable examples to illustrate the usability of the obtained main results. These results extend and generalize the corresponding results given in the existing literature. Moreover, we provide some applications on the existence and uniqueness of solutions for a certain type of nonlinear integral equations.
\end{abstract}

2010 Mathematics Subject Classification: 54A40; 03E72; 54H25

Keywords: fuzzy set, complex valued $t$-norm, complex valued fuzzy $b$-metric space, fixed point

\section{INTRODUCTION}

Fixed point theory plays a fundamental role in mathematics and applied sciences, such as optimization, mathematical models and economic theories. Also, this theory have been applied to show the existence and uniqueness of the solutions of differential equations, integral equations and many other branches of mathematics [6, 18, 19]. A basic result in fixed point theory is the Banach contraction principle. Since the appearance of this principle, there has been a lot of activity in this area.

In 2011, Azam et al. [4] defined the notion of a complex valued metric space which is more general than the well-known metric space and obtained some fixed point results for a pair of mappings satisfying a rational inequality. In this line, Rouzkard et al. [21] studied some common fixed point theorems in this space to generalize the result of [4]. Ahmad et al. [2] investigated some common fixed point results for the mappings satisfying rational expressions on a closed ball in such space. Later, Rao et al. [20] gave a common fixed point theorem in complex valued b-metric spaces, generalizing both the b-metric spaces introduced by Czerwik [5] and the complex valued metric spaces. After the establishment of this new idea, Mukhemier [14] presented common fixed point results of two self-mappings satisfying a rational inequality in complex valued $b$-metric spaces. Verma [26] studied common fixed point theorems 
using property (CLCS) in these spaces. In recent years, there has been a considerable literature on fixed point theory in complex valued metric spaces [1, 15, 16, 25].

In 1965, Zadeh [28] introduced the concept of a fuzzy set theory to deal with the unclear or inexplicit situations in daily life. Using this theory, Kramosil and Michalek [12] defined the concept of a fuzzy metric space. Grabiec [8] gave contractive mappings on a fuzzy metric space and extended fixed point theorems of Banach and Edelstein in such space. Successively, George and Veeramani [7] slightly modified the notion of a fuzzy metric space introduced by Kramosil and Michalek [12] and then obtained a Hausdorff topology and a first countable topology on it. In the light of the results given in [7], Sapena [22] gave some examples and properties of fuzzy metric spaces. Also, Shukla et al. [24] extended the concept of fuzzy metric space to complex valued fuzzy metric space and obtained some fixed point results in this space. In recent years, many researchers have improved and generalized fixed point results for various contractive mappings in fuzzy metric spaces [3,9,10,13,17,23,27].

In this paper, we introduce the concept of a complex valued fuzzy $b$-metric space, generalizing both the notion of a complex valued fuzzy metric space introduced by Shukla et al. [24] and the notion of a $b$-metric space. Then, we give the topology induced by this space and also study some properties about this topology such as Hausdorffness. Moreover, we present some fixed point theorems for contraction mappings in this more general class of fuzzy metric spaces. Finally, we investigate the applicability of the obtained results to integral equations and show a concrete example which illustrate the application part.

\section{PRELIMINARIES}

Consistent with Shukla, Rodriguez-Lopez and Abbas [24], the following definitions and results will be needed in what follows.

$\mathbb{C}$ denotes the complex number system over the field of real numbers. We set $P=\{(a, b): 0 \leq a<\infty, 0 \leq b<\infty\} \subset \mathbb{C}$. The elements $(0,0),(1,1) \in P$ are denoted by $\theta$ and $\ell$, respectively.

Define a partial ordering $\preceq$ on $\mathbb{C}$ by $c_{1} \preceq c_{2}$ (or, equivalently, $c_{2} \succeq c_{1}$ ) if and only if $c_{2}-c_{1} \in P$. We write $c_{1} \prec c_{2}$ (or, equivalently, $\left.c_{2} \succ c_{1}\right)$ to indicate $\operatorname{Re}\left(c_{1}\right)<\operatorname{Re}\left(c_{2}\right)$ and $\operatorname{Im}\left(c_{1}\right)<\operatorname{Im}\left(c_{2}\right)$ (see, also, [4]). The sequence $\left\{c_{n}\right\}$ in $\mathbb{C}$ is said to be monotonic with respect to $\preceq$ if either $c_{n} \preceq c_{n+1}$ for all $n \in \mathbb{N}$ or $c_{n+1} \preceq c_{n}$ for all $n \in \mathbb{N}$.

We define the closed unit complex interval by $I=\{(a, b): 0 \leq a \leq 1,0 \leq b \leq 1\}$, and the open unit complex interval by $I_{\theta}=\{(a, b): 0<a<1,0<b<1\}$. $P_{\theta}$ denotes the set $\{(a, b): 0<a<\infty, 0<b<\infty\}$. It is obvious that for $c_{1}, c_{2} \in \mathbb{C}, c_{1} \prec c_{2}$ if and only if $c_{2}-c_{1} \in P_{\theta}$.

For $A \subset \mathbb{C}$, if there exists an element $\inf A \in \mathbb{C}$ such that it is a lower bound of $A$, that is, $\inf A \preceq a$ for all $a \in A$ and $u \preceq \inf A$ for every lower bound $u \in \mathbb{C}$ of $A$, then $\inf A$ is called the greatest lower bound or infimum of $A$. Similarly, we define $\sup A$, the least upper bound or supremum of $A$, in usual manner. 
Remark 1 ([24]). Let $c_{n} \in P$ for all $n \in \mathbb{N}$. Then,

(i) If the sequence $\left\{c_{n}\right\}$ is monotonic with respect to $\preceq$ and there exists $\alpha, \beta \in$ $P$ such that $\alpha \preceq c_{n} \preceq \beta$, for all $n \in \mathbb{N}$, then there exists a $c \in P$ such that $\lim _{n \rightarrow \infty} c_{n}=c$.

(ii) Although the partial ordering $\preceq$ is not a linear (total) order on $\mathbb{C}$, the pair $(\mathbb{C}, \preceq)$ is a lattice.

(iii) If $S \subset \mathbb{C}$ is such that there exist $\alpha, \beta \in \mathbb{C}$ with $\alpha \preceq s \preceq \beta$ for all $s \in S$, then $\inf S$ and $\sup S$ both exist.

Remark 2 ([24]). Let $c_{n}, c_{n}^{\prime}, z \in P$, for all $n \in \mathbb{N}$. Then,

(i) If $c_{n} \preceq c_{n}^{\prime} \preceq \ell$ for all $n \in \mathbb{N}$ and $\lim _{n \rightarrow \infty} c_{n}=\ell$, then $\lim _{n \rightarrow \infty} c_{n}^{\prime}=\ell$.

(ii) If $c_{n} \preceq z$ for all $n \in \mathbb{N}$ and $\lim _{n \rightarrow \infty} c_{n}=c \in P$, then $c \preceq z$.

(iii) If $z \preceq c_{n}$ for all $n \in \mathbb{N}$ and $\lim _{n \rightarrow \infty} c_{n}=c \in P$, then $z \preceq c$.

Definition 1 ([24]). Let $\left\{c_{n}\right\}$ be a sequence in $P$. Then, the sequence $\left\{c_{n}\right\}$ is said to diverge to $\infty$ as $n \rightarrow \infty$, and we write $\lim _{n \rightarrow \infty} c_{n}=\infty$, if for all $c \in P$ there exists an $n_{0} \in \mathbb{N}$ such that $c \preceq c_{n}$ for all $n>n_{0}$.

Definition 2 ([24]). Let $X$ be a nonempty set. A complex fuzzy set $M$ is characterized by a mapping with domain $X$ and values in the closed unit complex interval I.

Definition 3 ([24]). A binary operation $*: I \times I \rightarrow I$ is called a complex valued $t$-norm if:

$\left(n_{1}\right) c_{1} * c_{2}=c_{2} * c_{1}$

$\left(n_{2}\right) c_{1} * c_{2} \preceq c_{3} * c_{4}$ whenever $c_{1} \preceq c_{3}, c_{2} \preceq c_{4}$;

$\left(n_{3}\right) c_{1} *\left(c_{2} * c_{3}\right)=\left(c_{1} * c_{2}\right) * c_{3}$

$\left(n_{4}\right) c * \theta=\theta, c * \ell=c$

for all $c, c_{1}, c_{2}, c_{3}, c_{4} \in I$.

Example 1 ([24]). Let the binary operations $*_{1}, *_{2}, *_{3}: I \times I \rightarrow I$ be defined, respectively, by

(1) $c_{1} *_{1} c_{2}=\left(a_{1} a_{2}, b_{1} b_{2}\right)$, for all $c_{1}=\left(a_{1}, b_{1}\right), c_{2}=\left(a_{2}, b_{2}\right) \in I$;

(2) $c_{1} *_{2} c_{2}=\left(\min \left\{a_{1}, a_{2}\right\}, \min \left\{b_{1}, b_{2}\right\}\right)$, for all $c_{1}=\left(a_{1}, b_{1}\right), c_{2}=\left(a_{2}, b_{2}\right) \in I$;

(3) $c_{1} *_{3} c_{2}=\left(\max \left\{a_{1}+a_{2}-1,0\right\}, \max \left\{b_{1}+b_{2}-1,0\right\}\right)$

for all $c_{1}=\left(a_{1}, b_{1}\right), c_{2}=\left(a_{2}, b_{2}\right) \in I$.

Then, $*_{1}, *_{2}$ and $*_{3}$ are complex valued $t$-norms.

Example 2 ([24]). Define $*_{4}: I \times I \rightarrow I$ as follows:

$$
c_{1} *_{4} c_{2}= \begin{cases}\left(a_{1}, b_{1}\right), & \text { if }\left(a_{2}, b_{2}\right)=\ell ; \\ \left(a_{2}, b_{2}\right), & \text { if }\left(a_{1}, b_{1}\right)=\ell ; \\ \theta, & \text { otherwise, }\end{cases}
$$

for all $c_{1}=\left(a_{1}, b_{1}\right), c_{2}=\left(a_{2}, b_{2}\right) \in I$. Then, $*_{4}$ is a complex valued $t$-norm. 
Definition 4 ([24]). Let $X$ be a nonempty set, $*$ a continuous complex valued $t$-norm and $M$ a complex fuzzy set on $X^{2} \times P_{\theta}$ satisfying the following conditions:

$\left(M_{1}\right) \theta \prec M(x, y, c)$;

$\left(M_{2}\right) M(x, y, c)=\ell$ for every $c \in P_{\theta}$ if and only if $x=y$;

$\left(M_{3}\right) M(x, y, c)=M(y, x, c)$;

$\left(M_{4}\right) M(x, y, c) * M\left(y, z, c^{\prime}\right) \preceq M\left(x, z, c+c^{\prime}\right)$;

$\left(M_{5}\right) M(x, y, \cdot): P_{\theta} \rightarrow I$ is continuous

for all $x, y, z \in X$ and $c, c^{\prime} \in P_{\theta}$.

Then, the triplet $(X, M, *)$ is called a complex valued fuzzy metric space and $M$ is called a complex valued fuzzy metric on $X$. A complex valued fuzzy metric can be thought of as the degree of nearness between two points of $X$ with respect to a complex parameter $c \in P_{\theta}$.

\section{ON COMPLEX VALUED FUZZY $b$-METRIC SPACES}

In this section, we present the notion of a complex valued fuzzy $b$-metric space and study some of its topological aspects which strengthen this concept.

Definition 5. Let $X$ be a nonempty set, $s \geq 1$ a given real number, $*$ a continuous complex valued $t$-norm and $M$ a complex fuzzy set on $X^{2} \times P_{\theta}$ satisfying the following conditions:

$\left(b M_{1}\right) \quad \theta \prec M(x, y, c)$;

$\left(b M_{2}\right) M(x, y, c)=\ell$ for every $c \in P_{\theta}$ if and only if $x=y$;

$\left(b M_{3}\right) M(x, y, c)=M(y, x, c)$;

$\left(b M_{4}\right) M(x, y, c) * M\left(y, z, c^{\prime}\right) \preceq M\left(x, z, s\left(c+c^{\prime}\right)\right)$;

$\left(b M_{5}\right) M(x, y, \cdot): P_{\theta} \rightarrow I$ is continuous

for all $x, y, z \in X$ and $c, c^{\prime} \in P_{\theta}$.

Then, the quadruple $(X, M, *, s)$ is called a complex valued fuzzy $b$-metric space and $M$ is called a complex valued fuzzy $b$-metric on $X$.

It is seen that the above definition coincides with that of the complex valued fuzzy metric when $s=1$. Thus, the class of the complex valued fuzzy $b$-metric spaces is larger than that of the complex valued fuzzy metric spaces, that is, every complex valued fuzzy metric space is a complex valued fuzzy $b$-metric space.

Now, we shall give the examples of complex valued fuzzy $b$-metric spaces induced by the $b$-metric spaces.

Example 3. Let $(X, d, s)$ be a $b$-metric space. Let us consider a complex fuzzy set $M: X^{2} \times P_{\theta} \rightarrow I$ such that

$$
M(x, y, c)=\frac{a \cdot b}{a b+d(x, y)} \ell
$$

where $c=(a, b) \in P_{\theta}$. Then, $\left(X, M, *_{2}, s\right)$ is a complex valued fuzzy $b$-metric space. 
Example 4. Let $(X, d, s)$ be a $b$-metric space. Define the mapping $M: X^{2} \times P_{\theta} \rightarrow I$ by

$$
M(x, y, c)=e^{-\frac{d(x, y)}{a+b} \ell}
$$

where $c=(a, b) \in P_{\theta}$. Then, $\left(X, M, *_{2}, 2 s\right)$ is a complex valued fuzzy $b$-metric space.

As shown in the following examples, every complex valued fuzzy $b$-metric space may not be induced by a $b$-metric space.

Example 5. Let $X=(3,+\infty)$ and let $M: X^{2} \times P_{\theta} \rightarrow I$ be defined by

$$
M(x, y, c)= \begin{cases}\ell, & \text { if } x=y \\ \left(\frac{1}{x}+\frac{1}{y}\right) \ell, & \text { if } x \neq y .\end{cases}
$$

Then, it is easy to see that $\left(X, M, *_{3}, s\right)$ is a complex valued fuzzy $b$-metric space. Moreover, there is not a $b$-metric $d$ on $X$ inducing the given complex valued fuzzy $b$-metric. by

Example 6. Let $X=(0,+\infty)$ be endowed with the mapping $M: X^{2} \times P_{\theta} \rightarrow I$ given

$$
M(x, y, c)= \begin{cases}\left(\frac{x}{y}\right)^{a} \ell, & \text { if } x \leq y \\ \left(\frac{y}{x}\right)^{a} \ell, & \text { if } y \leq x\end{cases}
$$

where $a>0$. Then, $\left(X, M, *_{1}, s\right)$ is a complex valued fuzzy $b$-metric space. Also, there is not a $b$-metric $d$ on $X$ inducing the given complex valued fuzzy $b$-metric.

Lemma 1. Let $(X, M, *, s)$ be a complex valued fuzzy $b$-metric space and $c_{1}, c_{2} \in \mathbb{C}$. If $c_{1} \prec c_{2}$, then $M\left(x, y, c_{1}\right) \preceq M\left(x, y, s c_{2}\right)$ for all $x, y \in X$.

Proof. Let us take $c_{1}, c_{2} \in P_{\theta}$ such that $c_{1} \prec c_{2}$. Therefore, $c_{2}-c_{1} \in P_{\theta}$ and so we have that for all $x, y \in X$

$$
M\left(x, y, c_{1}\right)=\ell * M\left(x, y, c_{1}\right)=M\left(x, x, c_{2}-c_{1}\right) * M\left(x, y, c_{1}\right) \preceq M\left(x, y, s c_{2}\right) .
$$

Let $(X, M, *, s)$ be a complex valued fuzzy $b$-metric space. An open ball $B_{M}(x, r, c)$ with center $x \in X$ and radius $r \in I_{\theta}, c \in P_{\theta}$ is defined by

$$
B_{M}(x, r, c)=\{y \in X: \ell-r \prec M(x, y, c)\} .
$$

Definition 6. Let $(X, M, *, s)$ be a complex valued fuzzy $b$-metric space. Then, $(X, M, *, s)$ is called a Hausdorff space if for any two distinct points $x, y \in X$, there exist two open balls $B\left(x, r_{1}, c_{1}\right)$ and $B\left(y, r_{2}, c_{2}\right)$ such that $B\left(x, r_{1}, c_{1}\right) \cap B\left(y, r_{2}, c_{2}\right)=\varnothing$.

Theorem 1. Every complex valued fuzzy b-metric space is a Hausdorff space.

Proof. Let $(X, M, *, s)$ be a complex valued fuzzy $b$-metric space and $x, y \in X$ with $x \neq y$. Then, we have $\theta \prec M(x, y, c) \prec \ell$. Taking $M(x, y, c)=r$, we obtain an $r_{1} \in I_{\theta}$ such that $r \prec r_{1} \prec \ell$. Therefore, there exists an $r_{2} \in I_{\theta}$ satisfying $r_{2} * r_{2} \succ r_{1}$. It is clear that $x \in B\left(x, \ell-r_{2}, \frac{c}{2 s}\right)$ and $y \in B\left(y, \ell-r_{2}, \frac{c}{2 s}\right)$. Also, we verify that $B\left(x, \ell-r_{2}, \frac{c}{2 s}\right) \cap$ 
$B\left(y, \ell-r_{2}, \frac{c}{2 s}\right)=\varnothing$. Suppose instead that there is a $z \in B\left(x, \ell-r_{2}, \frac{c}{2 s}\right) \cap B\left(y, \ell-r_{2}, \frac{c}{2 s}\right)$. Hence,

$$
r \prec r_{1} \prec r_{2} * r_{2} \prec M\left(x, z, \frac{c}{2 s}\right) * M\left(y, z, \frac{c}{2 s}\right) \preceq M(x, y, c)=r
$$

and so we get a contradiction.

Theorem 2. Let $(X, M, *, s)$ be a complex valued fuzzy b-metric space. Then, the family

$\tau_{M}=\left\{G \subseteq X:\right.$ for all $x \in G$, there exist $r \in I_{\theta}$ and $c \in P_{\theta}$ such that $\left.B_{M}(x, r, c) \subseteq G\right\}$ is a topology on $X$.

Proof. It is enough to show that if $G_{1}, G_{2} \in \tau_{M}$, then $G_{1} \cap G_{2} \in \tau_{M}$, since the other axioms are readily verified. Let $x \in G_{1} \cap G_{2}$. Then, there exist $r_{1}=\left(a_{1}, b_{1}\right), r_{2}=$ $\left(a_{2}, b_{2}\right) \in I_{\theta}$ and $c_{1}=\left(m_{1}, n_{1}\right), c_{2}=\left(m_{2}, n_{2}\right) \in P_{\theta}$ such that $B_{M}\left(x, r_{1}, c_{1}\right) \subseteq G_{1}$ and $B_{M}\left(x, r_{2}, c_{2}\right) \subseteq G_{2}$. Take

$$
r=\left(\min \left\{a_{1}, a_{2}\right\}, \min \left\{b_{1}, b_{2}\right\}\right) \text { and } c=\left(\min \left\{\frac{m_{1}}{s}, \frac{m_{2}}{s}\right\}, \min \left\{\frac{n_{1}}{s}, \frac{n_{2}}{s}\right\}\right) .
$$

It is clear that $r \in I_{\theta}$ and $c \in P_{\theta}$. Therefore, by applying Lemma 1 , we get $B(x, r, c) \subseteq$ $B\left(x, r, c_{1}\right)$ and $B(x, r, c) \subseteq B\left(x, r, c_{2}\right)$. Thus, we obtain $B(x, r, c) \subseteq G_{1} \cap G_{2}$, completing the proof.

Then, $\left(X, \tau_{M}\right)$ is called the topological space induced by the complex valued fuzzy $b$-metric space $(X, M, *, s)$.

Example 7. (i) The complex valued fuzzy $b$-metric space defined in Example 5 induces the discrete topological space on $X$ since for $x \in X, B_{M}(x, r, c)=\{x\}$ whenever $r_{1}=r_{2}<\frac{1}{3}-\frac{1}{x}$.

(ii) The complex valued fuzzy $b$-metric space defined in Example 6 induces the usual topological space on $X \subset \mathbb{R}$ because

$$
B_{M}(x, r, c)=\left(\max \left\{x\left(1-r_{1}\right)^{\frac{1}{a}}, x\left(1-r_{2}\right)^{\frac{1}{a}}\right\}, \min \left\{\frac{x}{\left(1-r_{1}\right)^{\frac{1}{a}}}, \frac{x}{\left(1-r_{2}\right)^{\frac{1}{a}}}\right\}\right)
$$

for $x \in X, r \in I_{\theta}$ and $c \in P_{\theta}$.

Proposition 1. Let $\left(X, M_{1}, *, s\right)$ and $\left(X, M_{2}, *, s\right)$ be two complex valued fuzzy $b$ metric spaces. Define the mappings $M: X^{2} \times P_{\theta} \rightarrow I$ and $N: X^{2} \times P_{\theta} \rightarrow I$ by

$$
M(x, y, c)=M_{1}(x, y, c) * M_{2}(x, y, c),
$$

and

$N(x, y, c)=\left(\min \left\{\operatorname{Re}\left(M_{1}(x, y, c)\right), \operatorname{Re}\left(M_{2}(x, y, c)\right)\right\}, \min \left\{\operatorname{Im}\left(M_{1}(x, y, c)\right), \operatorname{Im}\left(M_{2}(x, y, c)\right)\right\}\right)$.

Then, the following results hold:

(i) $(X, M, *, s)$ is a complex valued fuzzy $b$-metric space if $p * q \neq \theta$ with $p, q \neq \theta$.

(ii) $(X, N, *, s)$ is a complex valued fuzzy $b$-metric space.

(iii) $\tau_{M}=\tau_{N}$ 
Proof. (i) and (ii) are obvious.

(iii) Let $G \in \tau_{M}$. Then, for all $x \in G$, there exist an $r \in I_{\theta}$ and a $c \in P_{\theta}$ such that $B_{M}(x, r, c) \subseteq G$. Now, take an $r^{\prime} \in I_{\theta}$ with $\left(\ell-r^{\prime}\right) *\left(\ell-r^{\prime}\right) \succ \ell-r$. If $z \in B_{N}\left(x, r^{\prime}, c\right)$, then we have

$\ell-r^{\prime} \prec\left(\min \left\{\operatorname{Re}\left(M_{1}(x, z, c)\right), \operatorname{Re}\left(M_{2}(x, z, c)\right)\right\}, \min \left\{\operatorname{Im}\left(M_{1}(x, z, c)\right), \operatorname{Im}\left(M_{2}(x, z, c)\right)\right\}\right)$.

Therefore, from the fact that

$$
\ell-r \prec\left(\ell-r^{\prime}\right) *\left(\ell-r^{\prime}\right) \prec M_{1}(x, z, c) * M_{2}(x, z, c)=M(x, z, c)
$$

it follows that $z \in B_{M}(x, r, c)$. Thus, we infer that $G \in \tau_{N}$.

Conversely, let $G \in \tau_{N}$. Then, for all $x \in G$, there exist an $r \in I_{\theta}$ and a $c \in P_{\theta}$ such that $B_{N}(x, r, c) \subseteq G$. If $z \in B_{M}(x, r, c)$, then we have

$$
\ell-r \prec M(x, z, c)=M_{1}(x, z, c) * M_{2}(x, z, c) .
$$

Therefore, since $M_{1}(x, z, c) * M_{2}(x, z, c) \preceq M_{1}(x, z, c)$ and $M_{1}(x, z, c) * M_{2}(x, z, c) \preceq$ $M_{2}(x, z, c)$, we get $\ell-r \prec N(x, z, c)$. Thus, $z \in B_{N}(x, r, c)$ and this implies that $G \in \tau_{M}$

Let $(X, d, s)$ be a $b$-metric space and $\tau_{d}$ be a topology induced by the $b$-metric $d$ on $X$. Then, we shall show that the topology $\tau_{d}$ coincides with the topology $\tau_{M}$, where $(X, M, *, s)$ is deduced from the $b$-metric $d$.

Example 8. Consider Example 3. Then, we have $\tau_{M}=\tau_{d}$. Indeed, let $G \in \tau_{M}$. Then, for all $x \in G$, there exist an $r=\left(r_{1}, r_{2}\right) \in I_{\theta}$ and a $c=(a, b) \in P_{\theta}$ such that $B_{M}(x, r, c) \subseteq G$. Let us choose a positive number $h=\min \left\{\frac{a b r_{1}}{1-r_{1}}, \frac{a b r_{2}}{1-r_{2}}\right\}$. Therefore, we obtain $B_{d}(x, h) \subseteq G$, where $B_{d}(x, h)$ is an open ball with centre $x$ and radius $h$ for the $b$-metric $d$ and thus $G \in \tau_{d}$.

On the other hand, let $G \in \tau_{d}$. Then, for all $x \in G$, there exists a positive number $h$ such that $B_{d}(x, h) \subseteq G$. Let us now take an arbitrary $c=(a, b) \in P_{\theta}$ and an $r=\left(r_{1}, r_{2}\right)=\left(\frac{h}{a b+h}, \frac{h}{a b+h}\right) \in I_{\theta}$. Hence, we get $B_{M}(x, r, c) \subseteq G$ and so that $G \in \tau_{M}$.

Example 9. Let $\left(X, M, *_{2}, 2 s\right)$ be a complex valued fuzzy $b$-metric space defined in Example 4. Then, $\tau_{M}=\tau_{d}$. Indeed, if $G \in \tau_{M}$, then, for all $x \in G$, there exist an $r=\left(r_{1}, r_{2}\right) \in I_{\theta}$ and a $c=(a, b) \in P_{\theta}$ such that $B_{M}(x, r, c) \subseteq G$. Take a positive number $h=\min \left\{-(a+b) \operatorname{In}\left(1-r_{1}\right),-(a+b) \operatorname{In}\left(1-r_{2}\right)\right\}$. Clearly, $B_{d}(x, h) \subseteq G$ and this shows that $G \in \tau_{d}$.

For the reverse inclusion, let $G \in \tau_{d}$. Then, for all $x \in G$, there exists a positive number $h$ such that $B_{d}(x, h) \subseteq G$. Let us consider an arbitrary $c=(a, b) \in P_{\theta}$ and an $r=\left(r_{1}, r_{2}\right)=\left(1-e^{\frac{-h}{a+b}}, 1-e^{\frac{-h}{a+b}}\right) \in I_{\theta}$. Thus, it follows from $B_{M}(x, r, c) \subseteq G$ that $G \in \tau_{M}$.

Definition 7. Let $(X, M, *, s)$ be a complex valued fuzzy $b$-metric space. 
(i) A sequence $\left\{x_{n}\right\}$ in $X$ converges to $x \in X$ if for every $r \in I_{\theta}$ and every $c \in P_{\theta}$, there exists an $n_{0} \in \mathbb{N}$ such that, for all $n>n_{0}, \ell-r \prec M\left(x_{n}, x, c\right)$. We denote this by $\lim _{n \rightarrow \infty} x_{n}=x$.

(ii) A sequence $\left\{x_{n}\right\}$ in $X$ is said to be a Cauchy sequence in $(X, M, *, s)$ if for every $c \in P_{\theta}, \lim _{n \rightarrow \infty} \inf _{m>n} M\left(x_{n}, x_{m}, c\right)=\ell$.

(iii) $(X, M, *, s)$ is said to be a complete complex valued fuzzy $b$-metric space if for every Cauchy sequence $\left\{x_{n}\right\}$ in $(X, M, *, s)$, there exists an $x \in X$ such that $\lim _{n \rightarrow \infty} x_{n}=x$.

The proofs of the following lemmas follow along similar lines as in [24] and are therefore omitted.

Lemma 2. Let $(X, M, *, s)$ be a complex valued fuzzy b-metric space. A sequence $\left\{x_{n}\right\}$ in $X$ converges to $x \in X$ if and only if $\lim _{n \rightarrow \infty} M\left(x_{n}, x, c\right)=\ell$ holds for all $c \in P_{\theta}$.

Lemma 3. Let $(X, M, *, s)$ be a complex valued fuzzy b-metric space. A sequence $\left\{x_{n}\right\}$ in $X$ is a Cauchy sequence if and only if for every $r \in I_{\theta}$ and every $c \in P_{\theta}$, there exists an $n_{0} \in \mathbb{N}$ such that, for all $m, n>n_{0}, \ell-r \prec M\left(x_{n}, x_{m}, c\right)$.

Example 10. Let $X=[0,1] \times\{0\} \cup\{0\} \times[0,1]$ and let $d: X \times X \rightarrow \mathbb{C}$ be the mapping defined by

$$
\begin{aligned}
& d((x, 0),(y, 0))=(x-y)^{2}(\alpha, 1) \\
& d((0, x),(0, y))=(x-y)^{2}(1, \beta) \\
& d((x, 0),(0, y))=d((0, y),(x, 0))=\left(\alpha x^{2}+y^{2}, x^{2}+\beta y^{2}\right)
\end{aligned}
$$

where $\alpha, \beta$ are fixed nonnegative real constants satisfying $\alpha \neq \frac{1}{\beta}$. Then, $(X, d, s)$ is a complete complex valued $b$-metric space with $s \geq 2$. Moreover, we define

$$
M(u, v, c)=\frac{a b}{a b+|d(u, v)|} \ell
$$

for all $u, v \in X, c=(a, b) \in P_{\theta}$. Thus, one can check that $\left(X, M, *_{2}, s\right)$ is a complete complex valued fuzzy $b$-metric space.

It follows from the above example that a complete complex valued fuzzy $b$-metric space can be induced by a complete complex valued $b$-metric space.

Definition 8. Let $(X, M, *, s)$ be a complex valued fuzzy $b$-metric space, $f: X \rightarrow X$ be a mapping and $x \in X$. Then, the mapping $f$ is continuous at $x$ if for any sequence $\left\{x_{n}\right\}$ in $X, \lim _{n \rightarrow \infty} x_{n}=x$ implies $\lim _{n \rightarrow \infty} f x_{n}=f x$.

If $f$ is continuous at each point $x \in X$, then we say that $f$ is continuous on $X$.

\section{MAIN RESUlts}

Firstly, we prove the Banach Contraction Theorem in the setting of complex valued fuzzy $b$-metric space. 
Theorem 3. Let $(X, M, *, s)$ be a complete complex valued fuzzy b-metric space such that, for every sequence $\left\{c_{n}\right\}$ in $P_{\theta}$ with $\lim _{n \rightarrow \infty} c_{n}=\infty$, we have

$$
\lim _{n \rightarrow \infty} \inf _{y \in X} M\left(x, y, c_{n}\right)=\ell
$$

for all $x \in X$. Let $f: X \rightarrow X$ be a mapping satisfying

$$
M\left(f x, f y, \frac{\lambda c}{s}\right) \succeq M(x, y, c)
$$

for all $x, y \in X$ and $c \in P_{\theta}$, where $\lambda \in(0,1)$. Then, $f$ has a unique fixed point in $X$.

Proof. We start by an arbitrary $x_{0} \in X$ and generate a sequence $\left\{x_{n}\right\}$ in $X$ by the iterative process

$$
x_{n}=f x_{n-1} \text { for all } n \in \mathbb{N} .
$$

If $x_{n}=x_{n-1}$ for some $n \in \mathbb{N}$, then $x_{n}$ is a fixed point of $f$. Consequently, assume that $x_{n} \neq x_{n-1}$ for all $n \in \mathbb{N}$. Now, we will prove that $\left\{x_{n}\right\}$ is a Cauchy sequence in $X$. Define

$$
B_{n}=\left\{M\left(x_{n}, x_{m}, c\right): m>n\right\}
$$

for all $n \in \mathbb{N}$ and $c \in P_{\theta}$. Due to $\theta \prec M\left(x_{n}, x_{m}, c\right) \preceq \ell$, for all $m \in \mathbb{N}$ with $m>n$ and from Remark 1(iii), inf $B_{n}=\beta_{n}$ exists for all $n \in \mathbb{N}$. Applying Lemma 1 and (4.1), we get

$$
M\left(x_{n}, x_{m}, c\right) \preceq M\left(x_{n}, x_{m}, \frac{s c}{\lambda}\right) \preceq M\left(f x_{n}, f x_{m}, c\right)=M\left(x_{n+1}, x_{m+1}, c\right),
$$

for $c \in P_{\theta}$ and $m, n \in \mathbb{N}$ with $m>n$. So, from the fact that

$$
\theta \preceq \beta_{n} \preceq \beta_{n+1} \preceq \ell \text { for all } n \in \mathbb{N}
$$

it follows that $\left\{\beta_{n}\right\}$ is a monotonic sequence in $P$. Therefore, utilizing Remark 1(i), we have an $\ell_{0} \in P$ satisfying

$$
\lim _{n \rightarrow \infty} \beta_{n}=\ell_{0} .
$$

Now, by successive application of the contractive condition (4.1), we have

$$
\begin{aligned}
M\left(x_{n+1}, x_{m+1}, c\right) & \succeq M\left(x_{n}, x_{m}, \frac{s c}{\lambda}\right)=M\left(f x_{n-1}, f x_{m-1}, \frac{s c}{\lambda}\right) \\
& \succeq M\left(x_{n-1}, x_{m-1}, \frac{s^{2} c}{\lambda^{2}}\right)=M\left(f x_{n-2}, f x_{m-2}, \frac{s^{2} c}{\lambda^{2}}\right) \\
& \succeq M\left(x_{n-2}, x_{m-2}, \frac{s^{3} c}{\lambda^{3}}\right) \\
& \succeq \cdots \succeq M\left(x_{0}, x_{m-n}, \frac{s^{n+1} c}{\lambda^{n+1}}\right),
\end{aligned}
$$

for $c \in P_{\theta}$ and $m, n \in \mathbb{N}$ with $m>n$. Thus,

$$
\beta_{n+1}=\inf _{m>n} M\left(x_{n+1}, x_{m+1}, c\right) \succeq \inf _{m>n} M\left(x_{0}, x_{m-n}, \frac{s^{n+1} c}{\lambda^{n+1}}\right) \succeq \inf _{y \in X} M\left(x_{0}, y, \frac{s^{n+1} c}{\lambda^{n+1}}\right) .
$$


Since $\lim _{n \rightarrow \infty} \frac{s^{n+1} c}{\lambda^{n+1}}=\infty$, by using the hypothesis along with (4.3), we obtain

$$
\ell_{0} \succeq \lim _{n \rightarrow \infty} \inf _{y \in X} M\left(x_{0}, y, \frac{s^{n+1} c}{\lambda^{n+1}}\right)=\ell,
$$

which implies that $\ell_{0}=\ell$. Thus, $\left\{x_{n}\right\}$ is a Cauchy sequence in $X$.

Since $(X, M, *, s)$ is a complete complex valued fuzzy $b$-metric space, by Lemma 2, there exists a $p \in X$ such that for all $c \in P_{\theta}$,

$$
\lim _{n \rightarrow \infty} M\left(x_{n}, p, c\right)=\ell .
$$

Next, we will show that $p$ is the fixed point of $f$. Due to $\left(b M_{4}\right)$ and the contractive condition (4.1), we have

$$
\begin{aligned}
M(p, f p, c) & \succeq M\left(p, x_{n+1}, \frac{c}{2 s}\right) * M\left(x_{n+1}, f p, \frac{c}{2 s}\right) \\
& =M\left(p, x_{n+1}, \frac{c}{2 s}\right) * M\left(f x_{n}, f p, \frac{c}{2 s}\right) \\
& \succeq M\left(p, x_{n+1}, \frac{c}{2 s}\right) * M\left(x_{n}, p, \frac{c}{2 \lambda}\right),
\end{aligned}
$$

for any $c \in P_{\theta}$. Letting the limit as $n \rightarrow \infty$, by (4.4) and Remark 2(ii), we get $M(p, f p, c)=\ell$ for all $c \in P_{\theta}$, which gives $f p=p$.

To prove the uniqueness of the fixed point $p$, let $q$ be another fixed point of $f$, that is, there is a $c \in P_{\theta}$ with $M(p, q, c) \neq \ell$. From (4.1), we obtain that

$$
\begin{aligned}
M(p, q, c)=M(f p, f q, c) & \succeq M\left(p, q, \frac{s c}{\lambda}\right)=M\left(f p, f q, \frac{s c}{\lambda}\right) \\
& \succeq M\left(p, q, \frac{s^{2} c}{\lambda^{2}}\right) \\
& \vdots \\
& \succeq M\left(p, q, \frac{s^{n} c}{\lambda^{n}}\right) \\
& \succeq \inf _{y \in X} M\left(p, y, \frac{s^{n} c}{\lambda^{n}}\right),
\end{aligned}
$$

for all $n \in \mathbb{N}$. Hence, since $\lim _{n \rightarrow \infty} \frac{s^{n} c}{\lambda^{n}}=\infty$, the above inequality turns into

$$
M(p, q, c) \succeq \ell,
$$

which gives a contradiction. Thus, we conclude that the fixed point of $f$ is unique.

Now, we present an example which shows the superiority of our assertion.

Example 11. Let $X=[0,1]$ and let $M: X^{2} \times P_{\theta} \rightarrow I$ be defined by

$$
M(x, y, c)=\frac{a b}{a b+(x-y)^{2}} \ell
$$


where $c=(a, b) \in P_{\theta}$. Then, one can readily verify that $\left(X, M, *_{1}, s\right)$ is a complete complex valued fuzzy $b$-metric space with $s=2$. Moreover, following the same procedure as in Example 3.10 of [24], we conclude that for any sequence $\left\{c_{n}\right\}$ in $P_{\theta}$ with $\lim _{n \rightarrow \infty} c_{n}=\infty$, we have $\lim _{n \rightarrow \infty} \inf _{y \in X} M\left(x, y, c_{n}\right)=\ell$ for all $x \in X$.

Now, we define a mapping $f: X \rightarrow X$ such that $f x=\alpha x^{2}$, where $0<\alpha<\frac{1}{4}$. By a routine calculation, we see that

$$
M\left(f x, f y, \frac{\lambda c}{2}\right) \succeq M(x, y, c)
$$

for all $x, y \in X$ and $c \in P_{\theta}$, where $\lambda=4 \alpha \in(0,1)$. Hence, all the conditions of Theorem 3 are satisfied and 0 is the unique fixed point of $f$.

Next, we establish the following fixed point theorem that extends the Jungck's Theorem [11] to the setting of complex valued fuzzy $b$-metric spaces.

Theorem 4. Let $(X, M, *, s)$ be a complete complex valued fuzzy b-metric space such that, for every sequence $\left\{c_{n}\right\}$ in $P_{\theta}$ with $\lim _{n \rightarrow \infty} c_{n}=\infty$, we have

$$
\lim _{n \rightarrow \infty} \inf _{y \in X} M\left(x, y, c_{n}\right)=\ell
$$

for all $x \in X$ and $f, g: X \rightarrow X$ be two mappings satisfying the following conditions:

(i) $g(X) \subseteq f(X)$,

(ii) $f$ and $g$ commute on $X$,

(iii) $f$ is continuous on $X$,

(iv) $M\left(g x, g y, \frac{\lambda c}{s}\right) \succeq M(f x, f y, c)$ for all $x, y \in X$ and $c \in P_{\theta}$, where $\lambda \in(0,1)$.

Then, $f$ and $g$ have a unique common fixed point in $X$.

Proof. Let $x_{0} \in X$. Due to $g(X) \subseteq f(X)$, we can choose an $x_{1} \in X$ such that $g x_{0}=f x_{1}$. Continuing this process, we can choose an $x_{n} \in X$ such that $f x_{n}=g x_{n-1}$. Now, we shall show that the sequence $\left\{f x_{n}\right\}$ is a Cauchy sequence. For all $n \in \mathbb{N}$ and $c \in P_{\theta}$, we define

$$
B_{n}=\left\{M\left(f x_{n}, f x_{m}, c\right): m>n\right\} .
$$

Since $\theta \prec M\left(f x_{n}, f x_{m}, c\right) \preceq \ell$, for all $m \in \mathbb{N}$ with $m>n$ and from Remark 1(iii) it follows that inf $B_{n}=\beta_{n}$ exists for all $n \in \mathbb{N}$. For $c \in P_{\theta}$ and $m, n \in \mathbb{N}$ with $m>n$, we obtain, by Lemma 1 and the condition (iv),

$$
M\left(f x_{n}, f x_{m}, c\right) \preceq M\left(f x_{n}, f x_{m}, \frac{s c}{\lambda}\right) \preceq M\left(g x_{n}, g x_{m}, c\right)=M\left(f x_{n+1}, f x_{m+1}, c\right) .
$$

Therefore, due to

$$
\theta \preceq \beta_{n} \preceq \beta_{n+1} \preceq \ell \text { for all } n \in \mathbb{N},
$$

$\left\{\beta_{n}\right\}$ is a monotonic sequence in $P$. So, using Remark 1(i), there exists an $\ell_{0} \in P$ such that

$$
\lim _{n \rightarrow \infty} \beta_{n}=\ell_{0}
$$


For $c \in P_{\theta}$ and $m, n \in \mathbb{N}$ with $m>n$, by utilizing the condition (iv), we have

$$
\begin{aligned}
M\left(f x_{n+1}, f x_{m+1}, c\right) & =M\left(g x_{n}, g x_{m}, c\right) \\
& \succeq M\left(f x_{n}, f x_{m}, \frac{s c}{\lambda}\right)=M\left(g x_{n-1}, g x_{m-1}, \frac{s c}{\lambda}\right) \\
& \succeq M\left(f x_{n-1}, f x_{m-1}, \frac{s^{2} c}{\lambda^{2}}\right)=M\left(g x_{n-2}, g x_{m-2}, \frac{s^{2} c}{\lambda^{2}}\right) \\
& \succeq M\left(f x_{n-2}, f x_{m-2}, \frac{s^{3} c}{\lambda^{3}}\right) \\
& \succeq \cdots \succeq M\left(f x_{0}, f x_{m-n}, \frac{s^{n+1} c}{\lambda^{n+1}}\right),
\end{aligned}
$$

which gives

$$
\begin{aligned}
\beta_{n+1} & =\inf _{m>n} M\left(f x_{n+1}, f x_{m+1}, c\right) \\
& \succeq \inf _{m>n} M\left(f x_{0}, f x_{m-n}, \frac{s^{n+1} c}{\lambda^{n+1}}\right) \\
& \succeq \inf _{y \in X} M\left(f x_{0}, y, \frac{s^{n+1} c}{\lambda^{n+1}}\right) .
\end{aligned}
$$

Since $\lim _{n \rightarrow \infty} \frac{s^{n+1} c}{\lambda^{n+1}}=\infty$ and from the hypothesis along with (4.5) it follows that

$$
\ell_{0} \succeq \lim _{n \rightarrow \infty} \inf _{y \in X} M\left(f x_{0}, y, \frac{s^{n+1} c}{\lambda^{n+1}}\right)=\ell,
$$

which yields $\ell_{0}=\ell$. Thus, $\left\{f x_{n}\right\}$ is a Cauchy sequence in $X$.

By completeness of $X$ and Lemma 2, there exists a $p \in X$ such that

$$
\lim _{n \rightarrow \infty} f x_{n}=p .
$$

By the condition (iv), one can easily verify that continuity of $f$ implies continuity of $g$. Therefore, $\lim _{n \rightarrow \infty} g f x_{n}=g p$. Since $f$ and $g$ commute on $X$, we have $\lim _{n \rightarrow \infty} f g x_{n}=g p$. Moreover, we know that $\lim _{n \rightarrow \infty} g x_{n-1}=p$ and so we obtain $\lim _{n \rightarrow \infty} f g x_{n-1}=f p$. According to the uniqueness of limit, we get $f p=g p$ and therefore $f g p=g g p$.

Now, repeated use of the condition (iv) gives

$$
\begin{aligned}
M(g p, g g p, c) & \succeq M\left(f p, f g p, \frac{s c}{\lambda}\right)=M\left(g p, g g p, \frac{s c}{\lambda}\right) \\
& \succeq \cdots \succeq M\left(g p, g g p, \frac{s^{n} c}{\lambda^{n}}\right)=M\left(g p, f g p, \frac{s^{n} c}{\lambda^{n}}\right) \\
& \succeq \inf _{y \in X} M\left(g p, y, \frac{s^{n} c}{\lambda^{n}}\right) .
\end{aligned}
$$


On taking the limit $n \rightarrow \infty$ and applying the hypothesis we deduce $M(\operatorname{gp}, \operatorname{gg} p, c)=\ell$, which in turn implies that

$$
g g p=f g p=g p .
$$

That is, $g p$ is a common fixed point of $f$ and $g$.

Finally, we will investigate that such a point is unique. Let $g p$ and $q$ be two distinct common fixed points of $f$ and $g$. On using the condition (iv) with $x=g p$ and $y=q$, we find

$$
\begin{aligned}
& \ell \succeq M(g p, q, c)=M(g g p, g q, c) \\
& \succeq M\left(f g p, f q, \frac{s c}{\lambda}\right)=M\left(g p, q, \frac{s c}{\lambda}\right) \\
& \vdots \\
& \succeq M\left(g p, q, \frac{s^{n} c}{\lambda^{n}}\right) \\
& \succeq \inf _{y \in X} M\left(g p, y, \frac{s^{n} c}{\lambda^{n}}\right) .
\end{aligned}
$$

Hence, taking into account $\lim _{n \rightarrow \infty} \frac{s^{n} c}{\lambda^{n}}=\infty$, we conclude that $M(g p, q, c)=\ell$. Thus, $g p=q$, which completes the proof.

Now, we give the following example to illustrate the validity of Theorem 4.

Example 12. Let $X=[0,1]$. Define $M: X^{2} \times P_{\theta} \rightarrow I$ as follows:

$$
M(x, y, c)=e^{-\frac{(x-y)^{2}}{a+b} \ell,}
$$

where $c=(a, b) \in P_{\theta}$. Clearly, $\left(X, M, *_{2}, s\right)$ is a complete complex valued fuzzy $b$ metric space with $s=4$.

On the other hand, let $\lim _{n \rightarrow \infty} c_{n}=\infty$ for any sequence $\left\{c_{n}\right\}$ in $P_{\theta}$, where $c_{n}=$ $\left(a_{n}, b_{n}\right)$. From the fact that $(x-y)^{2} \leq 1$ for all $x, y \in X$ it follows that

$$
\inf _{y \in X} M\left(x, y, c_{n}\right)=\inf _{y \in X} e^{-\frac{(x-y)^{2}}{a_{n}+b_{n}}} \ell=e^{-\frac{\sup _{y} \in X(x-y)^{2}}{a_{n}+b_{n}}} \ell \succeq e^{-\frac{1}{a_{n}+b_{n}}} \ell .
$$

Therefore, we have

$$
\lim _{n \rightarrow \infty} \inf _{y \in X} M\left(x, y, c_{n}\right) \succeq \lim _{n \rightarrow \infty} e^{-\frac{1}{a_{n}+b_{n}} \ell}=\ell .
$$

Consider the mappings $f, g: X \rightarrow X$ given by

$$
f(x)=x \text { and } g(x)=\frac{x}{4}
$$

One can readily verify that $g(X) \subseteq f(X)$ and $f$ is continuous on $X$. Besides, $f$ and $g$ commute on $X$. Furthermore, it is easy to find that the condition (iv) holds for all $x, y \in[0,1]$ with $\lambda=\frac{1}{4} \in(0,1)$. 
Thus, all of the assumptions of Theorem 4 are fulfilled and $0 \in X$ is the unique common fixed point of the involved mappings $f$ and $g$.

Let $(X, M, *, s)$ be a complete complex valued fuzzy $b$-metric space. The contraction condition for the mapping $f: X \rightarrow X$ can be changed as follows:

$$
\ell-M(f x, f y, c) \preceq \lambda[\ell-M(x, y, c)]
$$

for all $x, y \in X$ and $c \in P_{\theta}$, where $\lambda \in[0,1)$.

Then, we demonstrate a fixed point result for this class of contraction, which is a new generalization of the Banach contraction principle.

Theorem 5. Let $(X, M, *, s)$ be a complete complex valued fuzzy b-metric space and $f: X \rightarrow X$ be a mapping satisfying the contraction condition (4.6). Then, $f$ has a unique fixed point in $X$.

Proof. Let $x_{0}$ be an arbitrary element of $X$. By induction, we can construct a sequence $\left\{x_{n}\right\}$ in $X$ such that $x_{n}=f x_{n-1}$ for all $n \in \mathbb{N}$. Following the proof of Theorem 3.1 in [24], we observe that the sequence $\left\{x_{n}\right\}$ is a Cauchy sequence in $X$ and converges to some $p \in X$. We shall show that $p$ is a fixed point of $f$. By the contractive condition (4.6), we have

$$
\ell-M\left(f x_{n}, f p, c\right) \preceq \lambda\left[\ell-M\left(x_{n}, p, c\right)\right]
$$

for all $n \in \mathbb{N}$ and $c \in P_{\theta}$. The above inequality shows that

$$
\ell(1-\lambda)+\lambda M\left(x_{n}, p, c\right) \preceq M\left(f x_{n}, f p, c\right)
$$

for all $n \in \mathbb{N}$ and $c \in P_{\theta}$. Therefore,

$$
\begin{aligned}
M(p, f p, c) & \succeq M\left(p, x_{n+1}, \frac{c}{2 s}\right) * M\left(x_{n+1}, f p, \frac{c}{2 s}\right) \\
& =M\left(p, x_{n+1}, \frac{c}{2 s}\right) * M\left(f x_{n}, f p, \frac{c}{2 s}\right),
\end{aligned}
$$

for any $c \in P_{\theta}$. Making the limit as $n \rightarrow \infty$, from (4.7) and Remark 2(ii), we deduce that $M(p, f p, c)=\ell$ for all $c \in P_{\theta}$, which yields $f p=p$.

To investigate the uniqueness of the fixed point of $f$, suppose that there exists another $q \in X$ such that $f(q)=q$. Then, there is a $c \in P_{\theta}$ satisfying $M(p, q, c) \neq \ell$. For this $c$, by virtue of (4.6), we have

$$
\ell-M(p, q, c)=\ell-M(f p, f q, c) \preceq \lambda[\ell-M(p, q, c)] .
$$

Since $M(p, q, c) \neq \ell$, we obtain $\operatorname{Re}(M(p, q, c)) \neq 1$ or $\operatorname{Im}(M(p, q, c)) \neq 1$. Let $\operatorname{Re}(M(p, q, c)) \neq 1$. Therefore, we get

$$
1-\operatorname{Re}(M(p, q, c)) \leq \lambda(1-\operatorname{Re}(M(p, q, c)))<1-\operatorname{Re}(M(p, q, c)),
$$

which leads to a contradiction. The other case is similar to this one and so we skip the details. Thus, $M(p, q, c)=\ell$ for all $c \in P_{\theta}$ and the proof is concluded.

The following example validates the aforesaid theorem. 
Example 13. Let $X=[0,1]$ and let $M: X^{2} \times P_{\theta} \rightarrow I$ be given by the rule

$$
M(x, y, c)=\ell-\frac{(x-y)^{2}}{1+a b} \ell
$$

where $c=(a, b) \in P_{\theta}$. Then, $\left(X, M, *_{4}, s\right)$ is a complete complex valued fuzzy $b$ metric space. Define the mapping

$$
f: X \rightarrow X, f x=\frac{x^{2}}{4} .
$$

Therefore, we have

$$
\frac{(f x-f y)^{2}}{1+a b} \ell \preceq \lambda\left(\frac{(x-y)^{2}}{1+a b} \ell\right),
$$

where $\lambda \in\left[\frac{1}{4}, 1\right)$. Hence, we conclude that (4.6) holds, so all the required hypotheses of Theorem 5 are satisfied, and thus we deduce the existence and uniqueness of the fixed point of $f$. Here, 0 is the unique fixed point of $f$.

Corollary 1. Let $(X, M, *, s)$ be a complete complex valued fuzzy b-metric space and let $f: X \rightarrow X$ be a mapping satisfying

$$
\ell-M\left(f^{n} x, f^{n} y, c\right) \preceq \lambda[\ell-M(x, y, c)]
$$

for all $x, y \in X$ and $c \in P_{\theta}$, where $\lambda \in[0,1)$. Then, $f$ has a unique fixed point in $X$ (Here, $f^{n}$ is the $n$th iterate of $f$ ).

Proof. By Theorem 5, we get a unique $x \in X$ such that $f^{n} x=x$. From the fact that $f^{n} f x=f f^{n} x=f x$ and from uniqueness, it follows that $f x=x$. This shows that $f$ has a unique fixed point in $X$.

\section{APPLICATIONS TO EXISTENCE OF SOLUTIONS OF INTEGRAL EQUATIONS}

In this section, we study the existence theorem for a solution of the following integral equation by using our main results in the previous section:

$$
x(t)=\vartheta(t)+\beta \int_{0}^{1} \xi(t, s) \varphi(s, x(s)) d s, t \in[0,1],
$$

where

(i) $\vartheta:[0,1] \rightarrow \mathbb{R}$ is continuous;

(ii) $\varphi:[0,1] \times \mathbb{R} \rightarrow \mathbb{R}$ is continuous, $\varphi(t, x) \geq 0$ and there exists a $\lambda \in[0,1)$ such that

$$
|\varphi(t, x)-\varphi(t, y)| \leq \lambda|x-y|
$$

for all $x, y \in \mathbb{R}$

(iii) $\xi:[0,1] \times[0,1] \rightarrow \mathbb{R}$ is continuous at $t \in[0,1]$ for all $s \in[0,1]$ and measurable at $s \in[0,1]$ for all $t \in[0,1]$. Also, $\xi(t, s) \geq 0$ and $\int_{0}^{1} \xi(t, s) d s \leq L$;

(iv) $\lambda^{2} L^{2} \beta^{2} \leq \frac{1}{2}$.

Now, we prove the following result. 
Theorem 6. Suppose that the conditions (i)-(iv) hold. Then, the integral equation (5.1) has one and only one solution in $C([0,1], \mathbb{R})$, where $C([0,1], \mathbb{R})$ is the set of all continuous real valued functions on $[0,1]$.

Proof. Let $X=C([0,1], \mathbb{R})$ and let us define a mapping $f: X \rightarrow X$ by

$$
f x(t)=\vartheta(t)+\beta \int_{0}^{1} \xi(t, s) \varphi(s, x(s)) d s
$$

for all $x \in X$ and for all $t \in[0,1]$. Now, we have to show that the mapping $f$ satisfies all conditions of Theorem 5. Define a mapping $M: X^{2} \times P_{\theta} \rightarrow I$ by

$$
M(x, y, c)=\ell-\sup _{t \in[0,1]} \frac{(x(t)-y(t))^{2}}{e^{a b}} \ell
$$

where $c=(a, b) \in P_{\theta}$. Clearly, $\left(X, M, *_{4}, s\right)$ is a complete complex valued fuzzy $b$ metric space.

Moreover, for all $x, y \in X$ and $t \in[0,1]$, we have

$$
\begin{aligned}
|f x(t)-f y(t)| & =\beta\left|\int_{0}^{1} \xi(t, s) \varphi(s, x(s))-\xi(t, s) \varphi(s, y(s)) d s\right| \\
& \leq \beta \int_{0}^{1} \xi(t, s)|\varphi(s, x(s))-\varphi(s, y(s))| d s \\
& \leq \beta \int_{0}^{1} \xi(t, s) \lambda|x(s)-y(s)| d s \\
& \leq \beta L \lambda \sup _{t \in[0,1]}|x(t)-y(t)| .
\end{aligned}
$$

From the fact that

$$
\sup _{t \in[0,1]}|f x(t)-f y(t)| \leq \beta L \lambda \sup _{t \in[0,1]}|x(t)-y(t)|
$$

it follows that

$$
\begin{aligned}
\sup _{t \in[0,1]} \frac{|f x(t)-f y(t)|^{2}}{e^{a b}} & \leq \beta^{2} L^{2} \lambda^{2} \sup _{t \in[0,1]} \frac{|x(t)-y(t)|^{2}}{e^{a b}} \\
& \leq \frac{1}{2} \sup _{t \in[0,1]} \frac{|x(t)-y(t)|^{2}}{e^{a b}} .
\end{aligned}
$$

This proves that the mapping $f$ satisfy the contractive condition (4.6) appearing in Theorem 5 , and hence $f$ has a unique fixed point in $C([0,1], \mathbb{R})$, that is, the integral equation (5.1) has a unique solution in $C([0,1], \mathbb{R})$.

Next, we give an example of an integral equation and establish the existence of its solutions by using Theorem 6 . 
Example 14. Consider the following integral equation

$$
x(t)=\frac{1}{1+t}+2 \int_{0}^{1} \frac{s^{2}}{t^{2}+2} \cdot \frac{|\cos x(s)|}{5 e^{s}} d s, t \in[0,1] .
$$

It is seen that the above equation is of the form (5.1), for

$$
\beta=2, \vartheta(t)=\frac{1}{1+t}, \xi(t, s)=\frac{s^{2}}{t^{2}+2}, \varphi(t, x)=\frac{|\cos x|}{5 e^{t}} .
$$

Clearly, the mapping $\varphi$ is continuous on $[0,1] \times \mathbb{R}$ and we get

$$
\begin{aligned}
|\varphi(t, x)-\varphi(t, y)| & =\frac{1}{5 e^{t}}|\cos x|-|\cos y| \mid \\
& \leq \frac{1}{5 e^{t}}|\cos x-\cos y| \\
& \leq \frac{1}{5}|\cos x-\cos y| \\
& \leq \frac{1}{5}|x-y|
\end{aligned}
$$

for all $x, y \in \mathbb{R}$. Therefore, $\varphi$ satisfies the condition (ii) of the integral equation (5.1) with $\lambda=\frac{1}{5}$. One can readily check that the mapping $\vartheta$ is continuous and in view of

$$
\int_{0}^{1} \xi(t, s) d s=\int_{0}^{1} \frac{s^{2}}{t^{2}+2} d s=\frac{1}{t^{2}+2} \cdot \frac{1}{3} \leq \frac{1}{6}=L,
$$

the mapping $\xi$ satisfies the condition (iii). Also, we have

$$
\lambda^{2} \beta^{2} L^{2} \leq \frac{1}{2}
$$

So, all the hypotheses (i)-(iv) are fulfilled. Thus, applying the Theorem 6, we conclude that the integral equation (5.2) has a unique solution in $C([0,1], \mathbb{R})$.

\section{ACKNOWLEDGEMENT}

The author sincerely thanks the editor and the referees for their helpful comments and suggestions.

\section{REFERENCES}

[1] M. Abbas, M. Arshad, and A. Azam, "Fixed points of asymptotically regular mappings in complex-valued metric spaces," Georgian Math. J., vol. 20, no. 2, pp. 213-221, 2013, doi: 10.1515/gmj-2013-0013.

[2] J. Ahmad, A. Azam, and S. Saejung, "Common fixed point results for contractive mappings in complex valued metric spaces," Fixed Point Theory Appl., vol. 2014, no. 1, p. 67, 2014, doi: 10.1186/1687-1812-2014-67.

[3] I. Altun and D. Turkoglu, "Some fixed point theorems on fuzzy metric spaces with implicit relations," Commun. Korean Math. Soc., vol. 23, no. 1, pp. 111-124, 2008. 
[4] A. Azam, B. Fisher, and M. Khan, "Common fixed point theorems in complex valued metric spaces," Numer. Funct. Anal. Optim., vol. 32, no. 3, pp. 243-253, 2011, doi: 10.1080/01630563.2011.533046.

[5] S. Czerwik, "Contraction mappings in b-metric spaces," Acta mathematica et informatica universitatis ostraviensis, vol. 1, no. 1, pp. 5-11, 1993.

[6] B. C. Dhage, "Condensing mappings and applications to existence theorems for common solution of differential equations," Bull. Korean Math. Soc., vol. 36, no. 3, pp. 565-578, 1999.

[7] A. George and P. Veeramani, "On some results of analysis for fuzzy metric spaces," Fuzzy Sets Syst., vol. 90, no. 3, pp. 365-368, 1997, doi: 10.1016/S0165-0114(96)00207-2.

[8] M. Grabiec, "Fixed points in fuzzy metric spaces," Fuzzy Sets Syst., vol. 27, no. 3, pp. 385-389, 1988, doi: 10.1016/0165-0114(88)90064-4.

[9] V. Gregori, J.-J. Miñana, and D. Miravet, "Extended fuzzy metrics and fixed point theorems," Mathematics, vol. 7, no. 3, p. 303, 2019, doi: 10.3390/math7030303.

[10] N. Hussain, P. Salimi, and V. Parvaneh, "Fixed point results for various contractions in parametric and fuzzy b-metric spaces," J. Nonlinear Sci. Appl., vol. 8, no. 5, pp. 719-739, 2015.

[11] G. Jungck, "Commuting maps and fixed points," Amer. Math. Monthly, vol. 83, pp. 261-263, 1976.

[12] I. Kramosil and J. Michálek, "Fuzzy metrics and statistical metric spaces," Kybernetika, vol. 11, no. 5, pp. 336-344, 1975.

[13] F. Mehmood, R. Ali, C. Ionescu, and T. Kamran, "Extended fuzzy b-metric spaces," J. Math. Anal., vol. 8, no. 6, pp. 124-131, 2017.

[14] A. A. Mukheimer, "Some common fixed point theorems in complex valued-metric spaces," Sci. World J., vol. 2014, 2014, doi: 10.1155/2014/587825.

[15] P. Murthy, B. Fisher, and R. Kewat, "Periodic points of rational inequality in a complex valued metric space," Filomat, vol. 31, no. 7, pp. 2143-2150, 2017, doi: 10.2298/FIL1707143M.

[16] H. Nashine, M. Imdad, and M. Hasan, "Common fixed point theorems under rational contractions in complex valued metric spaces," J. Nonlinear Sci. Appl., vol. 7, pp. 42-50, 2014.

[17] T. Oner, M. B. Kandemir, and B. Tanay, "Fuzzy cone metric spaces," J. Nonlinear Sci. Appl., vol. 8, no. 5, pp. 610-616, 2015.

[18] H. Pathak and B. Fisher, "Common fixed point theorems with applications in dynamic programming," Glas. Mat., vol. 31, pp. 321-328, 1996.

[19] H. Pathak, S. Mishra, and A. Kalinde, "Common fixed point theorems with applications to nonlinear integral equations,” Demonstratio Math., vol. 32, no. 3, pp. 547-564, 1999.

[20] K. Rao, P. R. Swamy, and J. R. Prasad, "A common fixed point theorem in complex valued $b$ metric spaces," Bull. Math. Statist. Res., vol. 1, no. 1, pp. 1-8, 2013.

[21] F. Rouzkard and M. Imdad, "Some common fixed point theorems on complex valued metric spaces," Comput. Math. Appl., vol. 64, no. 6, pp. 1866-1874, 2012, doi: 10.1016/j.camwa.2012.02.063.

[22] A. Sapena, "A contribution to the study of fuzzy metric spaces," Appl. Gen. Topol., vol. 2, no. 1, pp. 63-75, 2001.

[23] S. Shukla, D. Gopal, and W. Sintunavarat, "A new class of fuzzy contractive mappings and fixed point theorems,” Fuzzy Sets Syst., vol. 350, pp. 85-94, 2018, doi: 10.1016/j.fss.2018.02.010.

[24] S. Shukla, R. Rodriguez-Lopez, and M. Abbas, "Fixed point results for contractive mappings in complex valued fuzzy metric spaces," Fixed Point Theory, vol. 19, no. 2, pp. 751-774, 2018, doi: 10.24193/fpt-ro.2018.2.56.

[25] W. Sintunavarat, Y. J. Cho, and P. Kumam, "Urysohn integral equations approach by common fixed points in complex-valued metric spaces," Adv. Difference Equ., vol. 2013, no. 1, p. 49, 2013, doi: 10.1186/1687-1847-2013-49. 
[26] R. K. Verma, "Fixed point theorems using (clcs) property in complex valued $b$-metric spaces," Facta Univ. Ser. Math. Inform., vol. 32, no. 3, pp. 269-292, 2017, doi: 10.22190/FUMI1703269V.

[27] D. Wardowski, "Fuzzy contractive mappings and fixed points in fuzzy metric spaces," Fuzzy Sets Syst., vol. 222, pp. 108-114, 2013, doi: 10.1016/j.fss.2013.01.012.

[28] L. A. Zadeh, "Fuzzy sets," Inf. Control, vol. 8, no. 3, pp. 338-353, 1965.

Author's address

İzzettin Demir

Duzce University, Faculty of Science and Arts, Department of Mathematics, 81620 Duzce, Turkey

E-mail address: izzettindemireduzce.edu.tr 